\title{
Effectiveness of Group and Individual Formats of a Combined Motivational Interviewing and Cognitive Behavioral Treatment Program for Problem Gambling: A Randomized Controlled Trial
}

\author{
Tian P. S. Oei and Namrata Raylu \\ The University of Queensland, Brisbane, Australia \\ Leanne M. Casey \\ Griffith University, Brisbane, Australia
}

\begin{abstract}
Background: The study aimed to assess the effectiveness of group and individual formats of a combined motivational interviewing and cognitive behavioral treatment (CBT) program for problem gamblers (PGs) using a randomized controlled design. Method: One hundred and two PGs were randomly assigned to individual or group CBT conditions. Twenty-eight of these participants were randomly allocated to a 6-week waitlist control condition prior to receiving the designated treatment. Results: At post-treatment, there were significant improvements in all dependent variables (frequency and amount gambled, gambling urges, gambling cognitions, negative psychological states, and life satisfaction) for both the treatment conditions but not for the waitlist condition. Individuals that completed the individual (compared to group) treatment condition generally had higher effect sizes for gambling correlates. Therapeutic gains for gambling correlates were generally maintained at 6-month follow-up. Conclusions: A combined motivational interviewing and CBT program applied in group or individual format can improve PG behaviors, as well as gambling correlates.
\end{abstract}

Keywords: CBT, gambling, group, individual, treatment.

\section{Introduction}

Cognitive behavioral treatment (CBT) appears to be effective in treating problem gamblers (PGs) (Raylu and Oei, in press). In addition, CBT programs that include motivation-enhancing techniques can improve treatment retention (Raylu and Oei, in press).

Although studies with PGs have assessed CBT in individual and group therapy formats or compared components of CBT in the two formats, a direct comparison of CBT delivered in the two formats has only been reported in one randomized controlled study. Dowling, Smith and

Reprint requests to Tian P. S. Oei, School of Psychology, University Queensland, St Lucia, Brisbane, NSW 4072, Australia. E-mail: oei@psy.uq.edu.au An extended version is also available online in the table of contents for this issue: http://journals.cambridge.org/jid_BCP 
Thomas (2007) found that treatment outcomes of both formats were similar. However, those in the group condition did not produce better outcomes in relation to state anxiety and self-esteem compared to waitlist condition. Furthermore, at 6-month follow-up, more individuals from the group (i.e. 40\%) than the individual condition (i.e. 8\%) still met the diagnostic criteria for pathological gambling. This study, however, only included a small sample $(n=56)$ of female participants who were experiencing problems, mainly with electronic games. These limitations are important as gender differences have been found in gambling behaviors, cognitions, urges and psychological states of gamblers (Raylu and Oei, in press). Furthermore, different forms of gambling are related to gambling problems to a different extent (Raylu and Oei, in press).

Thus, this study aimed to assess the effectiveness of group and individual formats of a combined motivational interviewing and CBT program for PGs by compensating for the limitations of the Dowling et al.'s (2007) study. We hypothesized that group and individual CBT would be more effective than a control condition at post-treatment and at 6-month follow-up. Also, based on the findings that individual therapy produces slightly superior results compared to group therapy among PGs (e.g. Dowling et al., 2007; Echeburua, Baez and Fernandez-Montalvo, 1996), we also hypothesized that individual CBT would produce superior outcomes to group CBT.

\section{Method}

\section{Participants and procedures}

Participants were 102 Brisbane residents who volunteered to take part in the study. Participants were randomly assigned to a treatment (group or individual CBT) condition using a computer generated randomized number system. Twenty-eight of the 102 participants (14 from group condition and 14 from individual condition) were also randomly allocated to a 6-week waitlist condition. These individuals completed their designated treatment after the wait period.

Overall, 19 of the 28 participants successfully completed waitlist condition. Thirty-five participants completed the individual condition, while 29 participants completed the group condition. Twenty participants in each treatment condition completed the 6-month follow-up.

Compromise power analyses using G Power software showed that $n=20$ per condition was needed to produce desirable power of greater than .8 (using medium effect size $=0.5$ and beta/alpha ratio $=1$ ).

\section{Measures}

Variables significantly related to PGs (Raylu and Oei, in press) were assessed at pre-treatment, post-treatment, and 6-month follow-up. These included gambling cognitions (Gambling Related Cognitions Scale; Raylu and Oei, 2004a), gambling urges (Gambling Urge Scale; Raylu and Oei, 2004b), psychological states (Depression Anxiety Stress Scale-21; Lovibond and Lovibond, 1995), and life satisfaction (Satisfaction with Life Scale; Diener, Emmons, Larsen and Griffin, 1985). Participants also reported on the average amount gambled per day and the frequency of gambling (5-point ordinal scale - never, monthly or less, 2-4 times/week, 2-3 times/week, four or more times/week) on gaming machines, table games, betting on animals and other forms of gambling in the past 2 weeks. The authors devised these items. 


\section{Treatment}

The treatment provided involved a manualized CBT program to treat PGs (Raylu and Oei, in press). Individual treatment sessions were provided in two hour weekly sessions (two modules/session) for 6 weeks. Group treatment sessions $\left(2 \frac{1}{2}\right.$ hours long) were run for a 6-week period, once a week.

\section{Results}

\section{Changes in dependent variables for the waitlist condition}

Repeated measures General Linear Modeling (GLM) analyses showed that there were no significant differences between pre and post waitlist scores for any of the dependent variables. The effect sizes (partial $\eta^{2}$ ) between pre and post waitlist scores ranged from 0 to .06 for most dependent variables except psychological states. The effect size for psychological states was .22 (.17 using "last observation carried forward" method intent to treat analyses).

\section{Changes in dependent variables for the two treatment conditions at the three assessment points}

A series of $2 \times 3$ ( 2 treatment conditions and 3 assessment points) repeated GLM analyses) were conducted for each dependent variable. These analyses were repeated after intention to treat analyses were completed. Those in the individual and waitlist conditions were more likely to have a higher distribution of income $\left\{\chi^{2}(6, n=98)=18.40, p<.01\right\}$ and employed full time $\left\{\chi^{2}(4, n=99)=15.06, p<.01\right\}$ than individuals in the group condition. Since all group (differences between the two treatment conditions) and interaction (interaction patterns between the two treatment conditions and three assessment points) effects were non-significant, these were not entered as covariates in the analyses.

Results (see Table 1) showed only time effects (differences between the three assessment points) were significant. In relation to psychological states, there were significant differences only between pre-treatment scores and the other two assessment points, $F(2,70)=8.63, p<$ .001 . Similar results were found with intent to treat analyses, $F(2,182)=17.57, p<.001$. Similar time effects as found for psychological states were found for life satisfaction, $F(2,70)=$ $13.52, p<.001$ [with intention to treat analysis, $F(2,182)=22.59, p<.001$ ]. There were significant differences in gambling urge scores between all three assessment points, $F(2,74)=$ $8.65, p<.001$ except between pre-treatment and follow-up. However, using intent to treat analyses, differences were found between all three assessment points except between posttreatment and follow-up as predicted, $F(2,178)=19.39, p<.001$. For gambling cognitions, post-treatment and follow-up scores were significantly less than pre-treatment scores, $F(2,70)=39.34, p<.001$. Similar results were found using intent to treat analyses, $F(2,180)=$ $56.67, p<.001$. For the average amount spent gambling per day, non-significant time effects were found, $F(2,60)=2.77$, ns. However, using intent to treat analyses, significant differences were found only between pre-treatment scores and the other two assessment points, $F(2,172\}=$ $8.29, p<.001$. For the frequency of gambling, the post-treatment scores were significantly less than pre-treatment scores and the follow-up scores were significantly higher than the pre treatment scores, $F(2,62)=77.38, p<.001$. Using intent to treat analyses, the difference between pre-treatment and follow-up scores was not significant, $F(2,172\}=33.31, p<.001$. 
Table 1. Results of the GLM analyses

\begin{tabular}{|c|c|c|c|c|c|c|c|c|}
\hline & \multicolumn{4}{|c|}{ Without intent to treat analyses } & \multicolumn{4}{|c|}{ With intent to treat analyses } \\
\hline & $\begin{array}{l}\text { Pre treatment } \\
\qquad M(S D)\end{array}$ & $\begin{array}{l}\text { Post treatment } \\
\qquad M(S D)\end{array}$ & $\begin{array}{l}\text { 6-month follow-up } \\
\qquad M(S D)\end{array}$ & $\begin{array}{l}\text { Effect size } \\
\text { partial } \eta^{2}\end{array}$ & $\begin{array}{l}\text { Pre treatment } \\
\qquad M(S D)\end{array}$ & $\begin{array}{l}\text { Post treatment } \\
\qquad M(S D)\end{array}$ & $\begin{array}{l}\text { 6-monthn follow-up } \\
\qquad M(S D)\end{array}$ & $\begin{array}{l}\text { Effect size } \\
\text { partial } \eta^{2}\end{array}$ \\
\hline \multicolumn{9}{|c|}{ Frequency of gambling } \\
\hline Group & $5.47(2.15)$ & $2.24(2.88)$ & $7.06(2.68)$ & .73 & $5.59(2.18)$ & $3.50(2.90)$ & $5.36(2.97)$ & .50 \\
\hline Individual & $5.81(2.31)$ & $2.75(1.84)$ & $7.50(2.80)$ & .56 & $5.66(2.29)$ & $3.38(2.23)$ & $4.90(3.07)$ & .40 \\
\hline \multicolumn{9}{|c|}{ Average amount gambled per day* } \\
\hline Group & $182(141)$ & $79(127)$ & $106(108)$ & .33 & $246(244)$ & $118(150)$ & $130(141)$ & .18 \\
\hline Individual & 218(239) & $142(217)$ & $224(268)$ & .16 & $438(775)$ & $162(234)$ & $182(250)$ & .11 \\
\hline \multicolumn{9}{|c|}{ Gambling cognitions } \\
\hline Group & $84.30(21.27)$ & $44.65(26.41)$ & $58.70(27.36)$ & .56 & $85.48(21.14)$ & $60.14(31.69)$ & $66.83(29.44)$ & .39 \\
\hline Individual & $78.47(15.59)$ & $40.12(18.31)$ & $54.12(23.96)$ & .72 & $76.72(21.01)$ & $50.24(26.60)$ & $53.92(27.51)$ & .54 \\
\hline \multicolumn{9}{|c|}{ Gambling urges } \\
\hline Group & $17.95(8.97)$ & $12.15(7.52)$ & $15.50(8.98)$ & .24 & $20.37(10.44)$ & $15.80(10.48)$ & $17.26(10.63)$ & .19 \\
\hline Individual & 19.11(11.70) & $10.21(6.62)$ & $14.79(10.69)$ & .38 & $18.94(10.51)$ & $11.82(7.42)$ & $13.40(9.03)$ & 29 \\
\hline \multicolumn{9}{|c|}{ Psychological states } \\
\hline Group & $49.00(29.06)$ & $28.20(22.84)$ & $32.00(35.05)$ & .30 & $47.91(28.27)$ & $33.40(25.64)$ & $35.12(26.30)$ & .20 \\
\hline Individual & $45.42(26.01)$ & 26.71(27.74) & $35.76(26.84)$ & .36 & $42.28(26.62)$ & $30.60(26.81)$ & $32.36(25.36)$ & .23 \\
\hline \multicolumn{9}{|c|}{ Life satisfaction } \\
\hline Group & $13.84(8.05)$ & 19.11(7.13) & $17.00(8.47)$ & .38 & $13.23(6.48)$ & $16.09(7.04)$ & $15.16(7.34)$ & .26 \\
\hline Individual & $13.78(6.78)$ & $18.61(7.48)$ & $16.89(5.36)$ & .44 & $13.78(7.33)$ & $17.92(7.48)$ & $17.42(6.60)$ & .29 \\
\hline
\end{tabular}

Note: effect size compares pre and post-treatment data only.

*rounded to nearest dollar. 
Effect size. Effect sizes (partial $\eta^{2}$ ) between pre and post-treatment ranged from .24 to .73 (.17 to .50 with intent to treat analyses) for the group condition for most dependent variables. For the individual condition, all effect sizes ranged from .16 to .72 (.11 to .54 with intent to treat analyses).

Significant reliable change. Percentage of participants that improved their post-treatment scores compared to their pre-treatment scores for all dependent variables ranged from 71-93\% for the individual condition and $68-93 \%$ for the group condition. Significant reliable changes in scores from pre-treatment to post-treatment were obtained for all gambling correlates for both conditions except for life satisfaction, where only statistically significant changes were observed.

\section{Discussion}

The results of this study showed significant improvements in all dependent variables at posttreatment for both treatment conditions compared to the waitlist condition. Effect sizes for both the treatment conditions were generally above the cut-off for high effect size (i.e. 0.14) and higher than what were found for the control condition. Improvements were generally maintained for most of the assessed variables at 6-month follow-up. These results are consistent with previous studies that have shown that CBT or a combined motivational interviewing and CBT program can significantly improve PG symptoms and gambling correlates (Raylu and Oei, in press).

Our findings showed that both individual and group CBT were effective in the treatment of PG and its correlates. Group therapy is not only just cost effective, it also enables participants to learn from and support each other. Individual therapy may be more suitable for those who can afford therapy and those who prefer discussing life events on a one-to-one basis. Consistent to the results of other studies that have compared the two formats with PGs (i.e. Dowling et al., 2007; Echeburua et al., 1996), participants that completed the individual (compared to group) condition generally had higher effect sizes for gambling correlates. The one-to-one contact with therapists may have allowed extra practice of skills that the participants were having difficulty mastering.

Limitations of this study included a short follow-up period, predominantly Caucasian sample, volunteered participants, low treatment retention rates and a waitlist group not distinct from the two treatment conditions. Future studies need to assess the effectiveness of group and individual therapy both in clinical and controlled university settings, how gambling variables (e.g. gambling cognitions) and demographics influence relapse, personal factors (e.g. personality traits) that may influence the effectiveness of the two formats of therapy with PGs, and whether there are any differences in treatment outcomes between gamblers experiencing problems with different forms of gambling.

\section{References}

Diener, E., Emmons, R., Larsen, R. and Griffin, S. (1985). The satisfaction with life scale. Journal of Personality Assessment, 49, 71-75.

Dowling, N., Smith, D. and Thomas, T. (2007). A comparison of individual and group cognitivebehavioural treatment for female pathological gambling. Behaviour Research and Therapy, 45, 2192 2202. 
Echeburua, E., Baez, C. and Fernandez-Montalvo, J. (1996). Comparative effectiveness of three therapeutic modalities in the psychological treatment of pathological gambling: long-term outcome. Behavioural and Cognitive Psychotherapy, 24, 51-72.

Lovibond, S. H. and Lovibond, P. F. (1995). Manual for the Depression Anxiety Stress Scales (2nd Ed.). Sydney: Psychology Foundation.

Raylu, N. and Oei, T. P. S. (in press). A Cognitive Behavioral Therapy Program for Problem Gambling: therapists manual. Routledge.

Raylu, N. and Oei, T. P. S. (2004a). The Gambling Related Cognitions Scale (GRCS): development, confirmatory factor validation and psychometric properties. Addiction, 99, 757-769.

Raylu, N. and Oei, T. P. S. (2004b). The Gambling Urge Scale (GUS): development, confirmatory factor validation and psychometric properties. Psychology of Addictive Behaviors, 18, 100-105. 